\title{
Simultaneous onset of type 1 diabetes mellitus and silent thyroiditis under durvalumab treatment
}

\author{
Jose León Mengíbar1', Ismael Capel1, Teresa Bonfill2, Isabel Mazarico1, \\ Laia Casamitjana Espuña1 , Assumpta Caixàs ${ }^{1}$ and Mercedes Rigla1
}

'Endocrinology and Nutrition Department and 2Medical Oncology Department, Parc Taulí University Hospital, Sabadell, Barcelona, Spain
Correspondence should be addressed to J León Mengíbar Email

jleon_92@hotmail.com

\section{Summary}

Durvalumab, a human immunoglobulin G1 kappa monoclonal antibody that blocks the interaction of programmed cell death ligand 1 (PD-L1) with the PD-1 and CD80 (B7.1) molecules, is increasingly used in advanced neoplasias. Durvalumab use is associated with increased immune-related adverse events. We report a case of a 55-year-old man who presented to our emergency room with hyperglycaemia after receiving durvalumab for urothelial high-grade non-muscle-invasive bladder cancer. On presentation, he had polyuria, polyphagia, nausea and vomiting, and laboratory test revealed diabetic ketoacidosis (DKA). Other than durvalumab, no precipitating factors were identified. Pre-durvalumab blood glucose was normal. The patient responded to treatment with intravenous fluids, insulin and electrolyte replacement. Simultaneously, he presented a thyroid hormone pattern that evolved in 10 weeks from subclinical hyperthyroidism (initially attributed to iodinated contrast used in a previous computerised tomography) to overt hyperthyroidism and then to severe primary hypothyroidism (TSH: $34.40 \mu \mathrm{U} / \mathrm{mL}$, free thyroxine (FT4): $<0.23 \mathrm{ng} / \mathrm{dL}$ and free tri-iodothyronine (FT3): $0.57 \mathrm{pg} / \mathrm{mL}$ ). Replacement therapy with levothyroxine was initiated. Finally, he was tested positive for anti-glutamic acid decarboxylase (GAD65), anti-thyroglobulin (Tg) and antithyroid peroxidase (TPO) antibodies (Abs) and diagnosed with type 1 diabetes mellitus (DM) and silent thyroiditis caused by durvalumab. When durvalumab was stopped, he maintained the treatment of multiple daily insulin doses and levothyroxine. Clinicians need to be alerted about the development of endocrinopathies, such as DM, DKA and primary hypothyroidism in the patients receiving durvalumab.

\section{Learning points:}

- Patients treated with anti-PD-L1 should be screened for the most common immune-related adverse events (irAEs).

- Glucose levels and thyroid function should be monitored before and during the treatment.

- Durvalumab is mainly associated with thyroid and endocrine pancreas dysfunction.

- In the patients with significant autoimmune background, risk-benefit balance of antineoplastic immunotherapy should be accurately assessed.

\section{Background}

In recent years, immune checkpoint inhibitors (ICI) have emerged as effective therapies for advanced neoplasias and act as modulators of immune checkpoint proteins (1). ICI significantly improve the response rates and survival of patients with multiple neoplasms, including melanoma (2), non-small-cell lung cancer (NSCLC) (2) and renal cell carcinoma (RCC) (2). One of the most used pathways in oncological immunotherapy is the inhibition of PD1 protein (programmed cell death 1) by directly blocking its receptor (anti-PD1 molecules) or its binding 
to the PD1 ligand (anti-PD-L1). However, inhibition of the PD-1 pathway results in a reduction of 'selftolerance' with an apparent increase in immune-related adverse events (irAEs) (3). IrAEs affecting the endocrine system are the most frequent and complex toxicities and can evolve into life-threatening situations if not recognised. The most frequent endocrine irAE is thyroid dysfunction (2, 3), although other endocrinopathies, including hypophysitis, hypopituitarism $(2,3)$ and adrenal insufficiency (AI), have been described (4). Several authors have described cases of endocrinopathies caused by nivolumab, pembrolizumab and ipilimumab $(2,3,4$, 5). However, new molecules like durvalumab have been introduced in clinical practice in a short period of time, and its irAEs are not yet well known. The main indications (4) of durvalumab are classical Hodgkin's lymphoma (cHL), metastatic Merkel cell carcinoma (MMCC), RCC, NSCLC, squamous cell carcinoma of the head and neck, urothelial carcinoma, high microsatellite instability in tumour (MSI-H) and metastatic melanoma. Here we report a case with simultaneous onset of autoimmune primary hypothyroidism and autoimmune DM with diabetic ketoacidosis (DKA) under durvalumab treatment.

\section{Case presentation}

A 55-year-old Caucasian man presented to our emergency room with polyuria, polydipsia, polyphagia, nausea and vomiting in the last 2 weeks. Symptoms progressively deteriorated despite consuming a significant amount of fluids. He showed no fever or other infectious signs of disease.

The patient did not explain diarrhoea or malabsorption.

Three weeks before admission, he started a combination therapy of Bacillus Calmette-Guérin (BCG) and durvalumab according to a clinical trial protocol. He received one cycle of intravenous durvalumab at $10 \mathrm{mg} / \mathrm{kg}$ (1200 mg of durvalumab; weight, $120 \mathrm{~kg}$; body mass index, $\left.34.69 \mathrm{~kg} / \mathrm{m}^{2}\right)$.

He suffered from comorbidities like arterial hypertension and psoriasis. He received enalapril for arterial hypertension but not glucocorticoid therapy. A review of his family background revealed that his son was diagnosed with type $1 \mathrm{DM}$ and was positive for GAD65 Abs when he was 3 years old. There was no personal or family history of pancreatitis, thyroid dysfunction or other autoimmune disorders.

The patient was afebrile and normotensive with a pulse of $69 \mathrm{bpm}$, respiratory rate of 22 breaths per minute and oxygen saturation of $98 \%$ while breathing ambient air. On examination, he felt thirsty, tired, with dizziness. Physical examination and chest radiograph revealed normal findings.

\section{Case presentation for hypothyroidism}

One month before starting durvalumab treatment, routine blood test revealed an asymptomatic subclinical hyperthyroidism (TSH: $<0.01 \mu \mathrm{U} / \mathrm{mL}$; FT4: $1.41 \mathrm{ng} / \mathrm{dL}$ and FT3: $3.77 \mathrm{pg} / \mathrm{mL}$ )

The patient had previously undergone a computerised axial tomography (CAT scan) with iodinated radiographic contrast medium. Subclinical hyperthyroidism was considered secondary to the iodinated radiographic contrast medium, and no antithyroid drugs were introduced.

After durvalumab treatment and 2 weeks after presenting to our emergency room due to ketoacidosis, he manifested fatigue, constipation, muscle weakness and weight gain. Physical examination showed puffy face with under-eye oedema and dry skin. Palpation of the neck showed no goitre or cervical pain.

\section{Investigation}

Laboratory analyses showed hyperglycaemia $(417 \mathrm{mg} / \mathrm{dL})$ and increased anion gap metabolic acidosis $(\mathrm{pH}: 7.13$, $\mathrm{HCO}_{3}{ }^{-}$: $11.9 \mathrm{mEq} / \mathrm{L}$, anion gap: $21 \mathrm{mEq} / \mathrm{L}$ ) associated with ketosis (beta-hydroxybutyrate in capillary blood: $6.0 \mathrm{mmol} / \mathrm{L})$. Other metabolic derangements (hyponatraemia and hyperkalaemia) were related to DKA. Further blood test characterised glycaemic dysfunction as a type 1 DM (HbA1c: 8.4\%; C-peptide level: 0.02ng/mL; blood glucose level: $229 \mathrm{mg} / \mathrm{dL}$; GAD65: 22.31 U/mL and islet antigen-2 (IA2): 0.14 U/mL; Table 1).

Multiple fasting capillary glucose tests until 1 month prior to admission were normal (77-95 mg/dL). Abs for DM were not tested before starting durvalumab. Human leukocyte antigen typing was not checked.

Because the patient did not explain exocrine pancreatic disorders like diarrhoea or malabsorption, a deeper analysis of the exocrine pancreatic function was not performed.

Table 1 Laboratory data about glycaemic disorder.

\begin{tabular}{l} 
Variable \\
\hline Glucose (mg/dL) \\
Haemoglobin A1C (\%) \\
C-peptide (ng/mL) \\
GAD65 antibody (nmol/L) \\
IA2 antibody (U/mL)
\end{tabular}

\begin{tabular}{c} 
Reference range \\
\hline $70-110$ \\
$<6.5$ \\
$1.1-4.4$ \\
$<0.02$ \\
$<0.02$
\end{tabular}

\begin{tabular}{c}
\hline Result \\
\hline 417 \\
8.4 \\
0.02 \\
22.31 \\
0.14 \\
\hline
\end{tabular}

GAD65, glutamic acid decarboxylase; IA2, islet antigen 2 . 


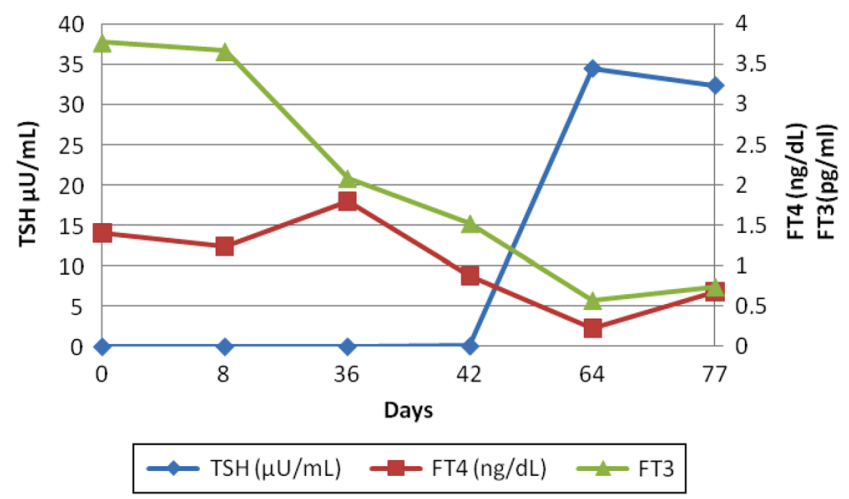

Figure 1

Thyroid function in a patient who developed silent thyroiditis under treatment with durvalumab.

\section{Investigation with hypothyroidism onset}

Laboratory analyses showed a rapid decrease in FT4 and increase in TSH (TSH: $0.08 \mu \mathrm{U} / \mathrm{mL}$, FT4: $0.88 \mathrm{ng} / \mathrm{dL}$ and FT3: $1.52 \mathrm{pg} / \mathrm{mL})$. AI was excluded by ACTH test $(250 \mathrm{mg}$ tetracosactide) that showed 0-min, 30-min and 60-min cortisol levels of $9.38 \mu \mathrm{g} / \mathrm{dL}, 23.70 \mu \mathrm{g} / \mathrm{dL}$ and $26 \mu \mathrm{g} / \mathrm{dL}$, respectively, and basal ACTH of $10.5 \mathrm{pg} / \mathrm{mL}$.

In the next 2 weeks, he developed primary hypothyroidism (TSH: $34.40 \mu \mathrm{U} / \mathrm{mL}, \quad$ FT4: $<0.23 \mathrm{ng} / \mathrm{dL}$ and FT3: $0.57 \mathrm{pg} / \mathrm{mL}$ ). Ordinary blood test showed positive antithyroid Abs: TSH receptor antibodies (TRAbs) $1.85 \mathrm{mUl} / \mathrm{mL}(0-1.75)$, TPO Abs $513 \mathrm{UI} / \mathrm{mL}(0-34)$ and $\mathrm{Tg}$ Abs 913 UI/mL (0-110; Fig. 1 and Table 2).

\section{Treatment}

The patient was diagnosed with DKA caused by new onset of type $1 \mathrm{DM}$ and primary autoimmune hypothyroidism, after a silent thyroiditis, secondary to durvalumab. He was treated with intravenous hydration, insulin drip with electrolyte replacement and levothyroxine. Levothyroxine treatment was initiated once AI was excluded. Thereafter, all the metabolic disturbances improved with resolution of hyperglycaemia, acidosis and undetectable FT4.

\section{Outcome and follow-up}

CT showed stable disease without progression. A new cycle of durvalumab was scheduled once the endocrine disorders were corrected. The patient continued to require daily multiple insulin injections (basal glargine, 60 U/day; prandial insulin aspart, $12 \mathrm{U} /$ meal) and levothyroxine $200 \mu \mathrm{g} / \mathrm{day}(1.6 \mu \mathrm{g} / \mathrm{kg})$ at his last follow-up, which was 3 months after hospital discharge.

\section{Discussion}

GAD65 Abs-positive, low C-peptide, sudden onset and persistence of hyperglycaemia with DKA confirmed type 1 DM. Low FT4, high TSH with TPOAbs, TGAbs and low levels of TRAbs-positive confirmed the diagnosis of primary autoimmune hypothyroidism. The causal relationship between durvalumab and development of autoimmune diabetes and hypothyroidism is reinforced by normal pretreatment fasting blood glucose levels and the preceding phase of silent thyroiditis with suppressed TSH, respectively. However, we cannot neglect the previous existence of GAD65 given the significant family history of type $1 \mathrm{DM}$ in a first-degree relative.

Endocrine disorders, especially thyroid dysfunction, are common irAE caused by ICI treatment (5). Endocrine disorders mostly have been related with nivolumab ( 2 , $3)$, pembrolizumab $(2,3,4,5)$ or ipilimumab $(2,3,4$, 5) treatment. To date, limited studies suggest endocrine disorders are caused by durvalumab.

The first report on durvalumab endocrine disorders was the PACIFIC (6) study. In this trial, durvalumab was used to treat stage III NSCLC in 1414 patients. Immunemediated hypothyroidism and hyperthyroidism occurred in 136 patients $(9.6 \%)$ and $81(5.7 \%)$, respectively. New onset of type $1 \mathrm{DM}$ without an alternative aetiology occurred in one patient $(<0.1 \%)$, AI occurred in $13(0.9 \%)$ and $\mathrm{AI}$ and diabetes insipidus caused by hypopituitarism occurred in one $(<0.1 \%)$.

Marchand et al. (7) describe a case of DM under durvalumab treatment. The patient developed DM 3 months after durvalumab treatment, but it was not

Table 2 Thyroid function in a patient who developed silent thyroiditis under treatment with durvalumab.

\begin{tabular}{|c|c|c|c|c|c|c|c|}
\hline & \multirow[b]{2}{*}{ Normal range } & \multicolumn{6}{|c|}{ Time (days) } \\
\hline & & 0 & 8 & 36 & 42 & 64 & 77 \\
\hline $\mathrm{TSH}(\mu \mathrm{U} / \mathrm{mL})$ & $0.4-4$ & $<0.01$ & $<0.01$ & $<0.01$ & 0.08 & 34.4 & 32.3 \\
\hline FT4 (ng/dL) & $0.8-1.8$ & 1.41 & 1.24 & 1.8 & 0.88 & 0.23 & 0.68 \\
\hline FT3 (pg/mL) & $2-4.4$ & 3.77 & 3.66 & 2.08 & 1.52 & 0.57 & 0.74 \\
\hline
\end{tabular}

FT3, free tri-iodothyronine; FT4, free thyroxine; TSH, thyrotropin. 
associated with positive autoantibodies. These authors described five cases of type $1 \mathrm{DM}$ in patients treated with nivolumab or pembrolizumab.

Way et al. (8) reported a case of an 84-year-old woman who after treatment with durvalumab developed DKA with low C-peptide $0.4 \mathrm{ng} / \mathrm{dL}$ (normal, $1.1-4.3 \mathrm{ng} / \mathrm{mL}$ ), glucose $142 \mathrm{mg} / \mathrm{dL}$ and positive GAD65 Abs. No other endocrine disorders were found.

The aetiology of PD1-1- and PDL-L1-induced diabetes is certainly of immune nature, but it is not related to the humoral immune dysfunction that characterises type 1 DM. This may be due to cell-mediated immune activation that occurs with PD-1 and PD-L1 therapies (9). In the revised case series, cell-mediated toxicity appears to spare the alpha-cells and exocrine pancreas in most patients. However, beta-cell destruction is so severe that most patients present with DKA and undetectable C-peptide with severe symptoms, including polyuria, polyphagia and weight loss. Other endocrine dysfunctions, like Hashimoto's hypothyroidism or AI, associated with PD1-1 and PDL-L1, are also associated with type 1 DM.

There are no biomarkers to predict the onset of these forms of endocrine disorders, so regular screening for early detection is mandatory (10). An in-depth screening for risk factors of autoimmune dysfunction, including personal or familial autoimmune illness like vitiligo, arthritis or autoimmune endocrinopathies may help in the rational selection of patients for this treatment. In some patients, it would be interesting to run antibody tests against the beta-cells and thyroid gland before starting the treatment.

For early detection, regular and frequent screening for dysglycaemia and thyroid or cortisol disorders is recommended. Baseline glycaemic parameters and thyroid function must be documented prior to immunotherapy in oncologic setting.

The potential appearance of autoimmune disorders with the use of ICI versus the appearance of other secondary effects using conventional chemotherapeutic drugs should be considered before choosing an appropriate oncologic therapy.

\section{Declaration of interest}

The authors declare that there is no conflict of interest that could be perceived as prejudicing the impartiality of the research reported.

\section{Funding}

This research did not receive any specific grant from any funding agency in the public, commercial or not-for-profit sector.

\section{Patient consent}

Written informed consent was obtained from the patient.

\section{Author contribution statement}

J L, I C, T B and L C treated the patient, reviewed the data and wrote the manuscript. I M, A C and M R reviewed the data and wrote the manuscript. The authors would like to thank Enago (www.enago.com) for the English language review.

\section{References}

1 Ribas A. Tumor immunotherapy directed at PD-1. New England Journal of Medicine 2012366 2517-2519. (https://doi.org/10.1056/ NEJMe1205943)

2 Brahmer JR, Tykodi SS, Chow LQM, Hwu WJ, Topalian SL, Hwu P, Drake CG, Camacho LH, Kauh J, Odunsi K, et al. Safety and activity of anti-PD-L1 antibody in patients with advanced cancer. New England Journal of Medicine 2012366 2455-2465. (https://doi. org/10.1056/NEJMoa1200694)

3 Kumar V, Chaudhary N, Garg M, Floudas CS, Soni P \& Chandra AB. Current diagnosis and management of immune related adverse events (irAEs) induced by immune checkpoint inhibitor therapy. Frontiers in Pharmacology 20178 49. (https://doi.org/10.3389/ fphar.2017.00049)

4 Cukier P, Santini FC, Scaranti M \& Hoff AO. Endocrine side effects of cancer immunotherapy. Endocrine-Related Cancer 201724 331-347. (https://doi.org/10.1530/ERC-17-0358)

5 Guaraldi F, La Selva R, Samà MT, D’Angelo V, Gori D, Fava P, Fierro MT, Savoia P \& Arva E. Characterization and implications of thyroid dysfunction induced by immune checkpoint inhibitors in real-life clinical practice: a long-term prospective study from a referral institution. Journal of Endocrinological Investigation 201841 549-556. (https://doi.org/10.1007/s40618-017-0772-1)

6 Antonia SJ, Villegas A, Daniel D, Vicente D, Murakami S, Hui R, Yokoi T, Chiappori A, Lee KH, de Wit M, et al. Durvalumab after chemoradiotherapy in stage III non-small-cell lung cancer. New England Journal of Medicine 2017377 1919-1929. (https://doi. org/10.1056/NEJMoa1709937)

7 Marchand L, Thivolet A, Dalle S, Chikh K, Reffet S, Vouillarmet J, Fabien N, Cugnet-Anceau C \& Thivolet C. Diabetes mellitus induced by PD-1 and PD-L1 inhibitors: description of pancreatic endocrine and exocrine phenotype. Acta Diabetologica 201956 441-448. (https://doi.org/10.1007/s00592-018-1234-8)

8 Way J, Drakaki A, Drexler A \& Freeby M. Anti-PD-L1 therapy and the onset of diabetes mellitus with positive pancreatic autoantibodies. BMJ Case Reports 2017 2017. (https://doi.org/10.1136/bcr-2017220415)

9 Sakurai K, Niitsuma S, Sato R, Takahashi K \& Arihara Z. Painless thyroiditis and fulminant type 1 diabetes mellitus in a patient treated with an immune checkpoint inhibitor, nivolumab. Tohoku Journal of Experimental Medicine 2018244 33-40. (https://doi. org/10.1620/tjem.244.33)

10 Kalra S. Post-immunotherapy new onset diabetes (PINOD) under recognized etiology, unexplored presentation. Annals of Translational Medicine 20186 84. (https://doi.org/10.21037/ atm.2018.10.71)

Received in final form 11 June 2019

Accepted 26 June 2019 